\title{
Parametric study on cohesive element for composite fuselage model
}

\begin{abstract}
In this paper, a numerical simulation on a new fabrication miniature composite fuselage structure- a woven composite laminated with an adhesively bonded butt joint under the axial compression loading is presented. A FEA via Abaqus/Explicit was utilized to capture the complete compressive responses to predict the crushing behaviour and its mechanical strength from the initial compression loading till its final failure mode. A woven C-glass fiber/epoxy $200 \mathrm{~g} / \mathrm{m} 2$ composite laminated [908] with the orthotropic elastic material properties is modeled as a continuum composite layup in the proposed numerical model. The adhesively bonded joint progression was modeled using the cohesive element technique. The proposed model was used to observe the crushing load and collapse modes under the axial compression impact. The finite element results showed a good agreement with the experimental results under the actual quasi-static tests. The validation code was extended to further undergo parametric studies in order to visualise the effect of angle orientations and special laminate cases as to attain the optimum design criteria for the proposed composite structure.
\end{abstract}

Keyword: C-glass composite; Cohesive element; FEA; Fuselage structure 